\title{
Ampullar ectopic pregnancy following ipsilateral partial isthmic salpingectomy
}

\author{
F. J. G. Kusters • D. M. J. Oom • A. C. Van Hof
}

Received: 2 July 2013 / Accepted: 23 April 2014 / Published online: 16 May 2014

(C) Springer-Verlag Berlin Heidelberg 2014

\begin{abstract}
The study is about ampullar ectopic pregnancy following ipsilateral partial isthmic salpingectomy. An ectopic pregnancy is any pregnancy outside of the uterine cavity. Pregnancies in the fallopian tube account for $97 \%$ of all ectopic pregnancies. Fifty-five percent of these pregnancies occur in the ampulla, $25 \%$ in the isthmus, $17 \%$ in the fimbriae, and $3 \%$ of the pregnancy is situated in the abdominal cavity, ovary, or uterine cervix. Diagnosing an ectopic pregnancy can be difficult; however, the widespread availability of pregnancy tests and the present high-resolution ultrasound possibilities have resulted in earlier diagnosis and have thus reduced the chance of massive intraabdominal bleeding and hypovolemic shock at presentation. We present a rare case of a recurrent ectopic pregnancy, occuring in the distal remnant of the ampulla of the fallopian tube, after a previous partial ipsilateral salpingectomy. This case shows that a strict adherence to the Dutch guideline would probably have led to an earlier detection.
\end{abstract}

Keywords Recurrent · Ectopic pregnancy

\section{Introduction}

An ectopic pregnancy is any pregnancy outside of the uterine cavity. Pregnancies in the fallopian tube account for $97 \%$ of all ectopic pregnancies. Fifty-five percent of these pregnancies occur in the ampulla, $25 \%$ in the isthmus, $17 \%$ in the fimbriae and $2-4 \%$ are interstitial pregnancy. In $3 \%$, the pregnancy is situated in the abdominal cavity, ovary or uterine cervix [1,2]. Risk factors for ectopic pregnancy include a previous ectopic pregnancy, antenatal DES-exposition, previous tubal surgery,

F. J. G. Kusters $(\bowtie) \cdot$ D. M. J. Oom • A. C. Van Hof

Department of Gynaecology, Ikazia Ziekenhuis Rotterdam,

Rotterdam, Netherlands

e-mail: fkusters@gmail.com salpingitis, infertility (including in vitro fertilisation treatment), smoking, pregnancy with intrauterine contraception device in situ and pregnancy after tubal ligation $[3,4]$. Since the $1980 \mathrm{~s}$, the incidence of ectopic pregnancies in the Netherlands has risen from 0.7 to $1 \%$ (as a percentage of live born infants). The most likely cause seems to be the rising number of patients with genital infections, in particular infections due to Chlamydia trachomatis [5]. Diagnosing an ectopic pregnancy can be difficult; however, the widespread availability of pregnancy tests and the present high-resolution ultrasound possibilities have resulted in earlier diagnosis and have thus reduced the chance of massive intra-abdominal bleeding and hypovolemic shock at presentation. The diagnosis is based on clinical suspicion in combination with ultrasonographical findings and serum human chorionic gonadotrophin levels. A suboptimal rise in serial serum human chorionic gonadotrophin (HCG) levels and the absence of a distinguishable intrauterine pregnancy after the discriminatory zone of a HCG titre of 2,000 IU/L has been reached are suggestive of the presence of an ectopic pregnancy. The Dutch guideline advises a diagnostic laparoscopy in any patient without an evident intrauterine gravidity and HCG levels $>2,000 \mathrm{IU} / \mathrm{L}$, or in patients with $\mathrm{HCG}$ levels $>1,500 \mathrm{IU} / \mathrm{L}$ with complaints of abdominal pain or with presence of free intra-abdominal fluids. We present a rare case of a recurrent ectopic pregnancy, occurring in the distal remnant of the ampulla of the fallopian tube, after a previous partial ipsilateral salpingectomy.

\section{Case report}

A 38-year-old female patient, gravid 9 parity 5, presented at the emergency room with lower abdominal pain and a positive pregnancy test, expected gestational age 8 weeks and 4 days. The medical history revealed five vaginal deliveries, two spontaneous miscarriages and a left-sided extrauterine pregnancy in the fallopian tube, treated with a laparoscopic 
salpingectomy in another hospital. Upon presentation, there was no vaginal blood loss. All vital signs were normal. Transvaginal ultrasonographic examination showed a small intrauterine fluid collection, suggestive of an early pregnancy. Also, a minor amount of free fluids in the pouch of Douglas was seen. No ectopic masses, especially on the right side, were visualised. HCG level was 2,970 IU/L. The differential diagnosis consisted of an early intrauterine pregnancy or an extrauterine pregnancy. Because of the small intrauterine fluid collection, the Dutch guideline was not adhered to, and she was admitted for observation and re-evaluation the next day.

The following day, the abdominal pain had mostly disappeared and there still was no vaginal blood loss. HCG levels dropped slightly to $2,720 \mathrm{IU} / \mathrm{L}$. As the patient improved clinically and no ectopic masses were visualised, she was released from the hospital with the probable diagnosis pending spontaneous abortion from a normal intrauterine pregnancy. Because an ectopic pregnancy could not be excluded, it was decided to evaluate her symptoms and HCG levels every 2 days. She was reviewed in the outpatient clinic.

Serial vaginal ultrasounds were performed and serum HCG levels were obtained. On the fourth day since first presentation, there was an increase in $\mathrm{HCG}$ to $5,343 \mathrm{IU} / \mathrm{L}$. Because still no amniotic sac, embryo or yolk sac could be visualised in the uterus, a diagnostic laparoscopy was performed.

At laparoscopy, a normal right fallopian tube was found. Due to prior partial removal of the left tube, the left ovary was covered in adhesions. After adhesiolysis, a distended ampullar remnant of the left fallopian tube was found. The proximal isthmic part of the tube was absent. A $360^{\circ}$ view of the abdomen showed no further abnormalities. No corpus luteum was seen in either ovary, although due to adhesions the left ovary could not be properly assessed.

The distal tubal remnant with its contents was surgically removed. Histological examination confirmed the ectopic pregnancy in the ampullar part of the fallopian tube. At the first postoperative day, the patient was discharged in good health from the hospital. Her postoperative recovery was uneventful.

\section{Discussion}

This case presents a rare finding of a recurrent ipsilateral tubal ectopic pregnancy after partial salpingectomy. This is only the eighth case reported in the literature [6-11].

In the literature, three possible explanations have been postulated for an embryo to nest in the distal tubal remnant.

One suggests that lumina persists in the interstitial portion and distal remnant of the fallopian tube, allowing communication between the endometrial and peritoneal cavities, and thus migration of spermatozoa or the fertilised egg from the endometrial cavity to the distal remnant of the left fallopian tube. Another, in our vision, more plausible explanation for our case is that this recurrent ectopic pregnancy was conceived following passage of spermatozoa through the intact right fallopian tube and the pouch of Douglas to the distal remnant of the left fallopian tube where the oocyt was present. The final option is transperitoneal embryo migration [6].

When salpingectomy is warranted, complete salpingectomy should be attempted to eliminate the risk of recurrent ipsilateral ectopic pregnancy. Partial salpingectomy should be considered a suboptimal treatment.

Based on this case report and other case reports described in the literature, gynaecologists should be aware of the possibility of a persistent distal remnant of the fallopian tube even if the patient history states a removal of the tube in a previous case of an ectopic pregnancy, and hence the possibility of a recurrent ipsilateral ectopic pregnancy. In this case, despite HCG levels $>2,000 \mathrm{IU} / \mathrm{L}$, we decided not to perform a laparoscopy because the fluid collection in the uterus was misinterpreted as a probable intrauterine gravidity. To gain certainty, follow up every 2 days was performed and the diagnosis of an extrauterine gravidity could be made in time.

This case shows that a strict adherence to the Dutch guideline could have led to an earlier detection.

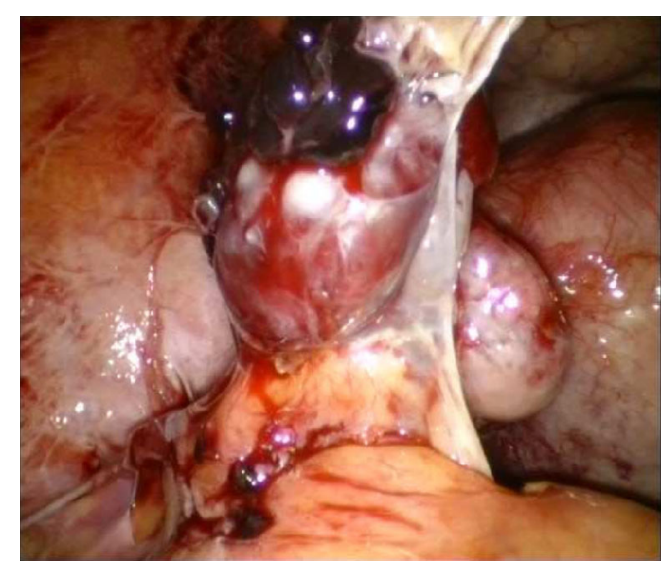

Conflict of interest FJG Kusters, DMJ Oom and AC Van Hof declare that they have no conflict of interest.

All procedures followed were in accordance with the ethical standards of the responsible committee on human experimentation (institutional and national) and with the Helsinki Declaration of 1975, as revised in 2000 (5). Informed consent was obtained from all patients for being included in the study.

\section{References}

1. Lozeau AM, Potter B (2005) Diagnosis and management of ectopic pregnancy. Am Fam Physician 72:1707-1714

2. Della-Giustina D, Denny M (2003) Ectopic pregnancy. Emerg Med Clin North Am 21:565-584 
3. Ankum WM, Mol BWJ, Bossuyt PMM, Van der Veen F (1996) Risk factors for ectopic pregnancy: a meta-analysis. Fertil Steril 65:10931099

4. Mol BWJ, Ankum WM, Bossuyt PMM, Van der Veen F (1995) Contraception and the risk for ectopic pregnancy. Contraception 52: 337-341

5. Egger M, Low N, Davey Smith G, Lindblom B, Herrman B (1998) Screening for chlamydial infections and the risk of ectopic pregnancy in a county in Sweden: ecological analysis. Br Med J 316:1766-1780

6. Liu YL, Hwang KS, Chu PW, Ding DC (2009) Recurrent ectopic pregnancy in the ipsilateral oviduct after prior partial salpingectomy. Taiwan J Obstet Gynecol 48(4):417-419
7. Zuzarte R, Khong CC (2005) Recurrent ectopic pregnancy following ipsilateral partial salpingectomy. Singap Med J 46(9):476-478

8. Chou SY, Hsu M, Chow P, Chiang HK, Su H, Hsu C (2009) Recurrent ipsilateral ectopic pregnancy after partial salpingectomy. Taiwan J Obstet Gynecol 48(4):420-421

9. Tan T, Elashry A, Tischner I, Jolaoso A (2007) Lightning does strike twice: recurrent ipsilateral tubal pregnancy following partial salpingectomy for ectopic pregnancy. J Obstet Gynaecol 27(5):534-535

10. Mathew M, Kumari R, Gowri V (2002) Three consecutive ipsilateral tubal pregnancies. Int J Gynaecol Obstet 78(2):163-164

11. Milingos DS, Black M, Bain C (2008) Three surgically managed ipsilateral spontaneous ectopic pregnancies. Obstet Gynecol 112(2Pt2):458-459 